\title{
RECIENTES PROGRESOS DE LA TERAPIA HORMONAL EN GINECOLOGIA*
}

\author{
Dr. Giorgio Hecht-Lucari**
}

El objeto de este trabajo es el de revisar cuanto hay de nuevo en la terapia hormonal en obstetricia y ginecología. Se trata de un objetivo ambicioso, dado que hoy es prácticamente imposible, no digo leer, sino hojear, todo lo publicado en la vastísima literatura mundial. Espero que no se me critique con severidad si no menciono este o aquel autor, 0 si desarrollo este singular tema de un modo no siempre homogéneo. A veces sucede que se da un particular realce a aquellos argumentos que más se conocen.

Me será imposible hablar de la terapia sin haber hablado antes del diagnóstico, en ausencia del cual una terapia dada no se habría desarrollado o no habría tenido posibilidad de éxito. No defino el concepto de nuevo y viejo en terapéutica: en el estudio de la historia se ha establecido que la época moderna comienza en la Revolución Francesa. En la terapéutica hormonal no existe un fenómeno de equivalente importancia que pueda servir como indicación del fin de una época y el comienzo de otra. Los tratamientos viejos se fusionan o se superponen frecuentemente con los nuevos, cuando no existe una integración feliz. Como siempre, el armamentario terapéutico del experto será mucho más vasto que el del colega menos experto y tal vez más moderno, pero no necesariamente adaptado a todas las eventualidades.

En obstetricia, un notable progreso diagnóstico no encuentra un equivalente progreso terapéutico. El propósito de la obstetricia es y ha sido siempre el dar a la madre un hijo vivo y sano. El estudio de la causa y de la profilaxis del "pregnancy wastage" (desperdicio del embarazo) constituye punto cardinal de esta rama de la medicina. No existe, a mi modo de ver, gran diferencia desde el punto de vista hormonal en la problemática de la interrupción del embarazo en el primer día o en los primeros meses, mientras que son mucho más complejos el conjunto de factores que pueden contribuir al parto prematuro y a la mortalidad peri-natal. Un problema, desde luego, domina todo el cuadro de la perspectiva del endocrinólogo: la gran ignorancia de los factores hormonales envueltos en el desarrollo fisiológico de la gravidez misma, desde el anidamiento del huevo hasta la ex-

\footnotetext{
* Trabajo presentado en el "Primer Centenario de la Universidad Nacional de Colombia".

* Docente libre de Clínica Obstétrica y Ginecológica de la Universidad de Roma.
} 
pulsión del producto de la concepción.

El escaso conocimiento del tema de la fisiología del embarazo es tan solo superado por el escaso conocimiento de los diversos cuadros patológicos. No sabemos aún por qué en la gravidez se producen cantidades así de colosales de gonadotropinas coriónicas, ni tenemos medidas sobre las funciones precisas de los estrógenos y de los progestágenos que, también ellos, alcanzan cerca del final del embarazo, valores diarios de verdadera consideración y bien superiores de aquellos que conocemos por fuera de la gravidez misma. Sólo hace poco hemos comprendido que el estriol representa gran parte de los estrógenos en la gravidez y no es como sucede fuera de la gestación, producto metabólico de la estrona y del beta estradiol sino sobre todo producto de la conversión del sulfato de dehidroisoandrosterona. El papel de la sulfoconjugación de los estrógenos en el embarazo debe, también, ser más dilucidado. Los actuales conocimientos, aunque fragmentarios, acerca de los estrógenos han traído, de todos modos, una notable contribución diagnóstica a la patología de la denominada "unidad feto-placentaria". De la dosificación del estriol en la orina, es posible extraer importantes deduc- ciones sobre el estado del feto y de la unidad feto-placenta. Con la determinación cuantitativa de un metabolito hormonal en la orina es posible obtener indicaciones importantes desde el punto de vista de la terapéutica quirúrgica. Desgraciadamente no es posible por ahora, influir con terapia médica sobre los procesos metabólicos mismos.

La aplicación al estudio de la gonadotropina coriónica de los modernos conocimientos en inmunología ha permitido lograr una serie de óptimos métodos para la determinación cualitativa y semicuantitativa de esta hormona en la orina y en el suero. Las consecuencias prácticas son obvias. En el conjunto se ha confirmado cuanto hemos aprendido antes de los más largos pero bastante precisos métodos biológicos. La determinación de la progesterona es todavía muy complicada para representar un método de rutina. El pregnandiol puede dosificarse con facilidad en la orina. Existen en verdad raros casos de embarazo interrumpido por un deficiente funcionamiento del cuerpo amarillo. Es presumible que en particular los casos de aborto espontáneo muy precoz, bien estudiados por Botella Llusiá en Madrid, puedan entrar en esta categoría. Pero, mejor aún, porque estos casos se pierden en la ma-

TABLA

EMBARAZOS EXITOSOS EN PACIENTES CON TRES O MAS ABORTOS PRIMARIOS RECURRENTES

\begin{tabular}{lcc}
\hline Tratamiento & $N^{0}$ de pacientes & Abortos \% \\
\hline Ninguno & 267 & 23 \\
Progesterona & 26 & 23 \\
Capronato de hidroxi-progesterona & 68 & 34 \\
Noretisterona & 31 & 45 \\
Dimetisterona & 30 & 27 \\
Acetato de medroxi-progesterona & 22 & 18 \\
\hline
\end{tabular}

Drug and Therapeutics Bulletin (4) 26, 1966 
sa de los abortos de causa "desconocida", la terapéutica con progesterona o con los progestágenos (progestativos sintéticos) no ha dado hasta ahora ningún resultado (tabla 1). No conocemos todavía ningún estudio que haya demostrado de modo estadísticamente válido un efecto de estos esteroides sobre el aborto habitual, en la amenaza de aborto o en el parto prematuro. El único dato positivo proviene de los estudios de Bengtsson, realizados en Lund. La inyección directa transabdominal, en el músculo uterino, de grandes dosis de 6-alfa-metil-17-alfa-acetoxiprogesterona ha producido sedación de la motilidad uterina. Nos podemos así preguntar cuántos de nuestros insucesos terapéuticos se imputan al hecho de que no es posible alcanzar en el receptor deseado una concentración adecuada del fármaco suministrado. El papel conjunto, parcial y total, que en el aborto y en el parto prematuro desempeñan las hormonas, el ambiente y el producto de la concepción; este último como tal, o como sede de la producción de las hormonas mismas, es tan difícil de aclarar, que nuestra oscuridad terapéutica no debe maravillarnos.

Como un progreso terapéutico magistral debe considerarse la síntesis de la ocitocina y la aplicación de esta sustancia, ya perfectamente dosificada y dosificable en la terapéutica obstétrica. Lo que se ha aprendido utilizando la ocitocina extractiva se ha podido perfeccionar, me parece fuera de duda, debido a que este polipéptido influye en la disminución de la patología perinatal.

Si este breve cuadro de la terapéutica hormonal en obstetricia no da motivo a excesivo optimismo, puede atribuirse también al modesto esfuerzo que por años se ha estado aplicando al estudio de la fisiología de la gravidez. No se podría decir que todo el progreso moderno de la bioquímica, de la electrónica, etc., haya sido aplicado de inmediato al estudio de la gravidez y con intensidad igual a aquella, de otras ramas de la medicina. Esta tendencia afortunadamente se ha modificado en los últimos años, por lo que es lícito un cauto optimismo para el futuro.

Un conjunto de factores, sobre todo socioeconómicos, ha contribuído a imprimirle un ritmo muy rápido al progreso en la terapia hormonal ginecológica. La fisiología y la fisiopatología de la reproducción, también importante en el campo veterinario, es una de las ramas de la medicina en las que más se hace y para la cual están hoy disponibles fondos notabilísimos para financiar la investigación. La convergencia de todos estos factores no podría menos que dar sus frutos, como ahora veremos.

Pende, Marañón y Roussy y Mosinger habían previsto que la hipófisis no podía, sola e independientemente tener una función predominante a manera de "director de orquesta de la sinfonía hormonal". Las relaciones anatomofisiológicas sobre el hipotálamo, aunque oscuras, no pueden ser ignoradas $\circ$ considerarse fortuitas. La demostración de la existencia de "releasing factors" (factores desencadenantes), ha confirmado hace poco la dependencia de la función antehipofisaria de los centros hipotalámi$\cos y$ hace esperar que en un futuro próximo estos factores puedan tener aplicación en el tratamiento de las insuficiencias hipofisarias por carencia de estimulación central.

El mecanismo de "feed back" (autorregulación) entre la antehipófisis y las glándulas endocrinas por ella controladas, es suficientemente conocido. Hoy debemos integrar estos 
viejos esquemas con autorregulación entre tales glándulas y el hipotálamo y con una pequeña autorregulación entre el hipotálamo y la hipófisis demostrado, en apariencia al menos, por el ACTH. De todas maneras parece aún válido el concepto de que entre el FSH, LH de un lado, y los estrógenos y la progesterona del otro, existe un equilibrio. Persiste una justificada incertidumbre sobre la forma como se verifica este equilibrio y sus relaciones cuantitativas entre estrógenos y progesterona necesarios para la ovulación. Parece justificada la hipótesis de que es necesaria una cierta actividad estrogénica a nivel del sistema hipofiso-hipotalámico para que se realice la liberación de aquel excedente de $\mathrm{LH}$ que es necesario para que se presente la ovulación. Hemos usado a propósito la expresión "actividad estrogénica" y no simplemente "estrógenos" porque la simple presencia de estrógenos se acompaña de la presencia simultánea de sustancias de actividad anti-estrogénica, por ejemplo la progesterona - ciertos progestágenos podrían ser insuficientes para producir el estímu- lo necesario para desencadenar la ovulación. Para prescindir de todas maneras de estas sutilezas fisiológicas, desde hace poco disponemos por primera vez en terapéutica de una sustancia capaz, en cierto número de casos, de influir la producción de gonadotropina por parte de la hipófisis anterior, cuando se ha perturbado la autorregulación: el clomifeno también conocido como clomid. Existe ahora abundante literatura sobre esta sustancia, cuyo mecanismo de acción no está por ahora aclarado por completo. En la experimentación animal, como también nosotros habíamos podido controlar, se trata de un fármaco a la vez "estrógeno" (levemente) y "antiestrogénico". Se puede suponer cuando se ha perturbado la autorregulación en su componente estrogénico, cuando falta el influjo rítmico de la cantidad creciente y menguante de los estrógenos ováricos sobre el centro, que el clomifeno pueda restablecer temporalmente este equilibrio, haciendo posible así una ovulación. Hace muy poco se ha puesto en duda la acción central del clomifeno $y$ se ha concluído en una

TABLA 2

RESPUESTA AL CITRATO DE CLOMIFENO EN PACIENTES CON VARIOS TRASTORNOS

\begin{tabular}{|c|c|c|c|}
\hline Trastornos & $\begin{array}{l}\text { Número de } \\
\text { Respondieron }\end{array}$ & $\begin{array}{l}\text { pacientes } \\
\text { Sin respuesta }\end{array}$ & Total \\
\hline Amenorrea primaria & 0 & 3 & 3 \\
\hline Síndrome de Ahumada del Castillo & 3 & 1 & 4 \\
\hline Síndrome de Chiari-Frommel & 0 & 1 & 1 \\
\hline Síndrome adrenogenital & 1 & 0 & 1 \\
\hline Amenorrea iatrogénica & 16 & 4 & 20 \\
\hline Enfermedad poliquística ovárica & 18 & 4 & 22 \\
\hline Déficit ovárico temprano & 0 & 3 & 3 \\
\hline Disfunción pituitárica primaria & 0 & 2 & 2 \\
\hline Desequilibrio ovárico-pituitario & 25 & 5 & 30 \\
\hline Oligomenorrea & 39 & 7 & 46 \\
\hline Ciclos irregulares & 20 & 8 & 28 \\
\hline Hemorragia disfuncional & 11 & 1 & 12 \\
\hline Ciclos normales (anovulatorios) & 3 & 2 & 5 \\
\hline Totales & 136 & 41 & 177 \\
\hline
\end{tabular}

Fertility and Sterility (17) 5, 1966. 
acción suya directa sobre el ovario, "condicionando" su respuesta a la gonadotropina. En espera de ulteriores pruebas de esta acción directa, perferimos abstenernos de cualquier comentario. Es verdad que mantenemos un cierto escepticismo, con base en cuanto se ha anotado en general sobre el tema del efecto directo de los estrógenos sobre el ovario humano. Algunos resultados con clomifeno se observan en las tablas 2 y 3 . Casos de esterilidad hasta ahora considerados intratables han sido resueltos con éxito.

\section{TABLA 3}

\section{RESUMEN DE LOS RESULTADOS OBTENIDOS CON CUATRO REGIMENES TERAPEUTICOS}

\begin{tabular}{lc}
\hline & $\begin{array}{c}\text { Ovulación aparente } \\
\text { por ciclo de } \\
\text { tratamiento }\end{array}$ \\
\hline Régimen de tratamiento: & \\
HGC & $0 / 26$ \\
HMG-clomifeno & $5 / 56$ \\
Clomifeno-HCG & $38 / 56$ \\
HMG-HCG & $19 / 20$ \\
\hline
\end{tabular}

Fertility and Sterility (17) 5, 1966.

Cuando la hipófisis es incapaz de producir gonadotropinas, es necesaria una terapia sustitutiva. No es del caso repetir el pro y el contra de la terapia con PMSG + HCG. Un progreso notabilísimo y sin igual representó la disponibilidad de gonadotropina humana con prevalente acción FSH. Existen dos fuentes; de un lado, la orina de la mujer en menopausia de la cual se extrae la HMUG (Human Menopausal Urinary Gonadotropin). Del otro, las hipófisis humanas de cadáveres de los cuales se extrae la HPG (Human Pituitary Gonadotropin). La primera fuente es desde luego la más rica; tan cierto es, que existen ya en el comercio dos preparados de HMUG. Se trata, como ya he dicho, no de FSH puro, si- no de un extracto de acción prevalentemente folículo-estimulante. En caso de amenorrea primitiva por insuficiencia hipofisaria e inclusive en pacientes hipofisectomizados, se han obtenido ovulaciones con subsecuentes embarazos cuando, previa maduración del folículo por intermedio del HMUG, se ha hecho seguir HCG (tabla 4). Imagino que sea conocido el hecho de que con esta terapia tenemos un alto porcentaje de superovulación con embarazos múltiples y consecuente interrupción espontánea de la gravidez. Recientemente Crooke de Birmingham ha informado que es posible evitar esas desagradables complicaciones (tabla 5). Este autor

\section{TABLA 5}

\section{INCREMENTO EN LAS DOSIS DE F. S. H. USA- DO EN TRATAMIENTOS SUCESIVOS HASTA OB- TENER UNA RESPUESTA POSITIVA}

\begin{tabular}{|c|c|c|}
\hline Incremento & $\begin{array}{l}\text { Hormona } \\
\text { U. I. }\end{array}$ & $\begin{array}{l}\text { folículo-estimulante } \\
1^{\text {a R. I. para }} \\
\text { F. S. H. (mg.)* }\end{array}$ \\
\hline 1 & 570 & 4.000 \\
\hline 2 & 710 & 5.000 \\
\hline 3 & 860 & 6.000 \\
\hline 4 & 1.140 & 8.000 \\
\hline 5 & 1.570 & 11.000 \\
\hline 6 & 2.140 & 15.000 \\
\hline 7 & 2.860 & 20.000 \\
\hline 8 & 4.290 & 30.000 \\
\hline
\end{tabular}

* Primera referencia internacional de la preparación de gonadotropina menopáusica humana. The Lancet, Sept. 3, 1966.

emplea una dosis mensual de FSH urinario, seguida al octavo día por una dosis de HCG. Si no se ha obtenido el resultado terapéutico deseado, al mes siguiente emplea una dosis mayor de FSH y así sucesivamente. No solo no se han hallado superovulaciones, sino que se ha evitado la hiper-estimulación del ovario. De paso, es interesante también subrayar cómo con esta gonadotropina huma- 
TABLA 4

RESULTADOS DE OVULACIONES CONTROLADAS EN 35 PACIENTES ESTERILES

\begin{tabular}{|c|c|c|c|c|c|c|c|c|c|c|}
\hline Pacientes con & $\begin{array}{c}\text { No de de } \\
\text { pacientes }\end{array}$ & $\begin{array}{l}\text { Excre } \\
\text { de g } \\
\text { Baja }\end{array}$ & $\begin{array}{c}\text { eción urin } \\
\text { onadotrop } \\
\text { Normal }\end{array}$ & $\begin{array}{l}\text { aria } \\
\text { inas } \\
\text { Alta }\end{array}$ & $\begin{array}{l}\text { Número } \\
\text { de trata- } \\
\text { mientos }\end{array}$ & $\begin{array}{l}\text { No de ovu- } \\
\text { laciones }\end{array}$ & $\begin{array}{l}\text { Número } \\
\text { de pacien- } \\
\text { test sin } \\
\text { ovulación }\end{array}$ & $\begin{array}{l}\text { Número } \\
\text { de em- } \\
\text { barazos }\end{array}$ & $\begin{array}{l}\text { No de na- } \\
\text { cimientos }\end{array}$ & $\begin{array}{l}\text { No de } \\
\text { abortos }\end{array}$ \\
\hline Amenorrea primaria & 3 & 3 & - & - & 4 & 4 & 0 & 2 & 3 & 0 \\
\hline Amenorrea secundaria & 22 & 15 & 6 & 1 & 41 & 24 & 4 & 14 & 16 & 3 \\
\hline Oligomenorrea & 7 & 6 & 1 & - & 14 & 12 & 0 & 2 & 2 & 0 \\
\hline Ciclos anovulatorios & 3 & - & 3 & - & 7 & 7 & 0 & 0 & 0 & 0 \\
\hline Total & 35 & 24 & 10 & 1 & 66 & 47 & 4 & 18 & 21 & 3 \\
\hline
\end{tabular}

GEMZELL C.: Experiences with human pituitary gonadotropins. Ovulation. 1966, Ed. Greenblatt. 
na se han obtenido resultados muy satisfactorios en algunos tipos de azoospermia.

$Y$ ahora, un breve comentario sobre la tiroides, pero no para decir del empleo de la hormona tiroidiana en terapéutica ginecológica. Ahora se ha puesto en evidencia en el cuerpo tiroides una nueva hormona, la tireocalcitonina, con la cual aparentemente es posible incluir de modo positivo la disposición ósea del calcio o por lo menos detener la descalcificación ósea. Existen algunos estudios que parecen indicar que la tireocalcitonina podría convertirse un día en un importante fármaco en la terapia de la osteoporosis, y por tal razón no quisimos excluirla de este breve repaso.

La terapia esteroide en ginecología ha hecho progresos gigantescos. Hablaré primero de la terapéutica con estrógenos, andrógenos y corticoides, después de ios progestágenos, con una breve revisión final a la terapia antiestrogénica y antiandrogénica en sentido lato. Aún en este tiempo un autor escribía que en conjunto los más frecuentes objetivos terapéuticos de la esteroideoterapia, consisten en: 1. La inducción de una metrorragia. 2. En la supresión de una metrorragia y 3 . En la regularización del ciclo. Numéricamente se trata de indicaciones muy importantes que, empero representan solo un síntoma. Es indudable que la plétora de esteroides hoy disponibles para la terapéutica de las disfunciones endocrinas ginecológicas, hace posible un tratamiento diferencial y no solo sintomático. La escogencia del esteroide o de la sucesión o combinación de los esteroides, dependerá de la seguridad del diagnóstico. En nuestra opinión esta terapia ha llegado hoy al grado de satisfacer objetivos más ambicio- sos que la simple eliminación más - menos transitoria de un síntoma. Podríamos decir, sin temor de ser contradichos, que se han podido disminuir los casos en los que es absolutamente necesario el tratamiento quirúrgico. En fin, es posible utilizar con limitaciones, como método de diagnóstico, el empleo sensato de los esteroides hormonales, cuando no se dispone de laboratorios en particular provistos: basta recordar el diagnóstico precoz del embarazo con las asociaciones estroprogestagénicas, el diagnóstico de receptividad del endometrio a los estrógenos o de simple falta de progesterona en la amenorrea secundaria, etc.

A los escritos de los doctores $\mathrm{Ha}$ ller y Lauritzen debemos dos recientes y óptimos aciertos de la moderna estrogenoterapia. Por lo demás, el empleo de tales hormonas viene hoy limitado a los casos en los cuales existen fundamentos fisiopatológicos lógicos para la aplicación terapéutica (tabla 6). Como principal excepción quiero citar la terapia de los disturbios de la menopausia. No fue posible hasta ahora, demostrar una correlación entre las características de estos disturbios y el déficit estrogénico, en tanto - si se considera el criterio "exjuvantibus" del éxito terapéutico- es imposible negar un papel patogénico al déficit de estrógenos. Una importante indicación de los estrógenos, la rápida hemostasia en la hemorragia disfuncional, ha perdido mucho de su importancia debido a la introducción en la terapéutica de los progestágenos. Lo mismo se dice para su empleo en el tratamiento de la dismenorrea de base disfuncional, dolencia en la cual hoy se obtiene la supresión de la ovulación con los contraceptivos orales en los que se suministra el 6-dehidro-retroprogesterona. 
TABLA 6

DIVERSOS EFECTOS DE ESTROGENOS EN MEDICINA HUMANA

\begin{tabular}{cc}
\hline Efectos estrogénicos & Deficiencia estrogénica o manifestaciones \\
de carencia
\end{tabular}

Estimulación del crecimiento de los órganos genitales

Proliferación del endometrio

Desarrollo de las mamas

Estimulación del epitelio vaginal, piel y mucosas

Influencia sobre centros vegetativos hipotalámicos, incremento del tono parasimpático.

Regulación de la secreción de gonadotropina

Estimulación de la actividad osteoblástica*

Retención de Ca y P.*

Regulación del nivel de colesterina y lipoproteínas (efecto protector de los vasos)

Mantenimiento del balance nitrogenado*
Atrofia del útero y anexos

Menopausia

Involución de las mamas

Atrofia del epitelio vaginal, craurosis vulvar y vaginal, flacidez de la piel

Síntomas vegetativos (con frecuencia distonía ergotropo-simpaticotónica)

Secreción y excreción incrementada de gonadotropina

Osteoporosis

Balance negativo de $\mathrm{Ca}$ y $\mathrm{P}$

Favorecimiento de arterioesclerosis e infarto de miocardio**

Balance nitrogenado negativo, debilidad muscular, pérdida del tono de los esfínteres.

HALLER J.: Indikationen zur Ostrogentherapie. Fortschr. Med. 84: № 18, 1966.

* No solo efecto de estrógenos.

** Relación causal discutida.

Los andrógenos tuvieron su momento glorioso en terapéutica ginecológica alrededor de 1950 gracias a la influencia de la escuela francesa. Su empleo queda hoy limitado al tratamiento de cierta forma de frigidez y en los disturbios de la menopausia, en combinación con los estrógenos. Recuerdo solo el cáncer de la glándula mamaria, terapia en la cual no tengo experiencia personal. Es difícil decir si la terapéutica con estrógenos en la menopausia suplantará del todo a la combinación estrógenos + andrógenos. Sin duda, la combinación posee algunos inconvenientes, sobre todo si se prolonga mucho o si se encuentra uno de los raros sujetos sensibles a los andrógenos. De otro lado como ya tuve ocasión de hacer resaltar años ha, existe entre las dos sustancias un sinergismo y un antagonismo que en ciertos casos puede representar ventajas terapéuticas con respecto al estrógeno solo. Por ejemplo, la asociación del efecto mineral-anabólico y prótido-anabólico del andrógeno con el mineral-anabólico más notorio del estrógeno en la osteoporosis. También aquí se deberá diferenciar en la escogencia del fármaco.

Los corticoides son interesantes en el tratamiento del síndrome adrenogenital y de una serie no bien definible, de límites vagos de disfunciones anovulatorias que Zener define "hiperfunción suprarrenal andrógena con infertilidad", en la que en ocasiones se han estado informando éxitos. La casuística es por lo demás modesta en su número y los casos no bien claros desde el punto de vis- 
TABLA 7

RESULTADOS EN EL TRATAMIENTO DE VIRILISMO Y OTROS DISTURBIOS

\begin{tabular}{|c|c|c|c|c|c|c|}
\hline Síntomas & $\begin{array}{l}\text { Cortril } \\
\text { Mejoría }\end{array}$ & 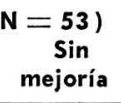 & $\begin{array}{l}\text { Delalutin } \\
\text { Mejoría }\end{array}$ & $\begin{array}{c}(N=83) \\
\text { Sin } \\
\text { mejoría }\end{array}$ & $\begin{array}{l}\text { Kenacort } \\
\text { Mejoría }\end{array}$ & $\begin{array}{c}(N=86) \\
\substack{\text { Sin } \\
\text { mejoría }}\end{array}$ \\
\hline Acné & 22 & & 23 & 2 & 29 & \\
\hline Hirsutismo & 11 & & 19 & & 32 & 1 \\
\hline Anovulación & 25 & & 44 & 3 * & 43 & \\
\hline Irregularidades menstruales & 8 & 1 & 34 & $4^{*}$ & 36 & \\
\hline Amenorrea & 6 & & 3 & $3^{*}$ & 4 & 1 \\
\hline Metabolismo general & 8 & & 3 & & 10 & \\
\hline Total & 80 & 1 & 142 & $12\left(10^{*}\right)$ & 154 & 2 \\
\hline
\end{tabular}

* Respondieron a Kenacort.

Pituitary ovarian endocrinology, Ed. Dorfman, 1963.

ta diagnóstico. En presencia de una anovulación sintomatológica masculinizante $y$ en ausencia de tumores masculinizantes, parece justo hacer una tentativa terapéutica en este sentido (tabla 7). En el acné, se ha empleado también la combinación: supresión de la ovulación y de la función ovárica - supresión suprarrenal- con buenos resultados, aunque transitorios (Ferin, com. personal).

En mi concepto, para desarrollar en forma correcta el tema de los progestágenos sería necesaria una relación aparte, dado que se trata del más grande progreso terapéutico en el ámbito de la terapia hormonal en ginecología. No es, sin embargo, escaso de interés, anotar que la progesterona había desilusionado la terapéutica ginecológica, tal vez porque debía administrarse por vía intramuscular, o porque en base a su rápido metabolismo, tenía una duración de acción muy breve. La introducción y el desarrollo de preparados a base de suspensiones microcristalinas de esta hormona, especialmente gracias a Kaufmann y a su escuela, representó un paso adelante no indiferente del punto de vista de la eficacia, aunque no de la vía de administración.
Es sin lugar a duda mérito de las hormonas corticoides sintéticas haber demostrado que es posible modificar la molécula de una hormona esteroide natural, sin que por ello deba resultar un esteroide inactivo.

Antes, no es raro que se obtengan sustancias de alta actividad hormonal, aunque no del todo equivalentes a la hormona natural. $Y$ es aquí donde reside el núcleo del problema de los progestágenos, junto con su gran valor en terapia ginecológica: se trata de un grupo $-y$ no estamos seguros aún en últimas- de esteroides hormonales diferentes el uno del otro y también diferentes de la progesterona en su espectro de acción. $Y$ es solamente en la terapia muy prolongada, en especial en asociación a un estrógeno, que la diferencia entre los varios progestágenos $y$ en parte las diferencias entre los progestágenos derivados de la 19-nor-testosterona o de la 17-alfa hidroxiprogesterona se vuelven muy vagas. En las terapias breves $y$, en su género, en el tratamiento con solo progestágenos, en ausencia de un estrógeno, existen diferencias sustanciales. El armamentario terapéutico debe tener más de un progestágeno, según la indicación. El fin fundamental de la terapia será 
la sustitución o el estímulo o la inhibición o el aprovechamiento de las propiedades farmacodinámicas. Se empleará entonces el progestágeno más adecuado y por una duración óptima, esta última a su vez adecuada al objetivo de la curación. Se puede así disfrutar al máximo de las propiedades intrínsecas de los esteroides en particular: la "progesteronosímil", la estrogénica, la antiestrogénica, la androgénica o la anti-androgénica, presentes en variedad en las diferentes moléculas.

En las tablas 8 y 9 hemos resumido, a manera de ejemplo, las relaciones existentes entre las indicaciones y la duración del tratamiento, de manera muy esquemática, además las relaciones entre el fin de la terapia y el tipo de indicación.

Es en extremo difícil, si no imposible, querer establecer breves normas generales sobre la escogencia del progestágeno. En concreto se puede de-

\section{TABLA 8}

DURACION DEL TRATAMIENTO

CON COMBINACIONES DE ESTROGENOS $Y$ GESTAGENOS (ORAL)

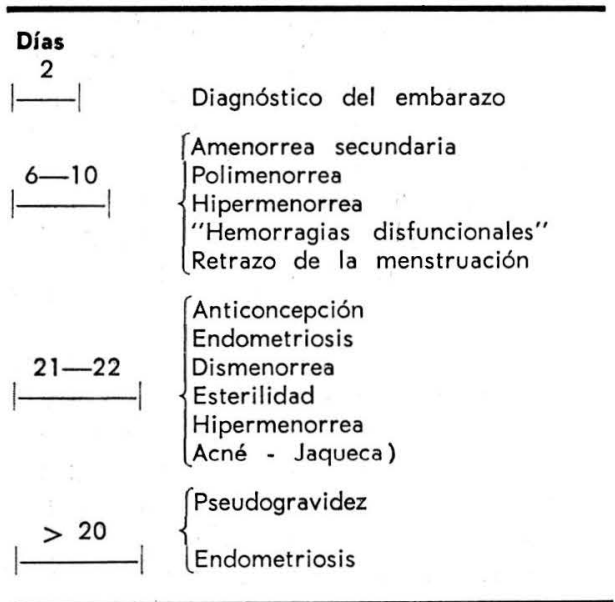

\section{TABLA 9}

\section{OBJETIVOS DE LA HORMONOTERAPIA}

(Gestágenos: Ejemplos)

\begin{tabular}{|c|c|}
\hline Sustitución & $\left\{\begin{array}{l}\text { Insuficiencia luteínica } \\
\text { Hipomenorrea }\end{array}\right.$ \\
\hline Estimulación & $\left\{\begin{array}{l}\text { Indirecta ("rebound effect") } \\
\text { tras suspender el tratamien- } \\
\text { to (tras inhibición) } \\
\text { Amenorrea secundaria }\end{array}\right.$ \\
\hline Represión & $\left\{\begin{array}{l}\text { Inhibición de la ovulación } \\
\text { Endometriosis } \\
\text { (indirecto) }\end{array}\right.$ \\
\hline $\begin{array}{l}\text { Efecto farma- } \\
\text { codinámico }\end{array}$ & $\left\{\begin{array}{l}\text { Endometriosis (directo) } \\
\text { Hemostasis (en hemorragias } \\
\text { disfuncionales) }\end{array}\right.$ \\
\hline
\end{tabular}

cir que para la terapia sustitutiva se deberán preferir más los "progestagenosímiles" mientras que para la terapéutica de estímulo de tipo indirecto ("rebound effect") se hará útil el empleo de combinaciones estroprogestágenas, para poder así aprovechar el efecto primario inhibidor de la asociación; lo mismo vale para la terapia inhibidora. La propiedad farmacodinámica más interesante en terapéutica ginecológica, la poseen los derivados de la 19-nor-testosterona, aunque hoy día por lo general también aquí se hace amplio uso de la combinación estro-progestágena (por ejemplo, endometriosis, metrorragia disfuncional, etc).

Tales combinaciones encuentran su mayor empleo en la contracepción, argumento tratado por el profesor Haller. Séame, de todos modos, concedida una observación. El tratamiento prolongado - como se usa en la contracepción - produce realmente en la mujer una serie de respuestas funcionales del todo comparables a las que se observan en el embarazo y no siempre del tipo presente, como con frecuencia se lee, en los prime- 
ros meses del embarazo (tabla 10). Por ejemplo, la dilatación ureteral es un fenómeno gravídico más bien tardío. Así también, la tolerancia disminuída a un recargo de glucosa, etc. No existen, sin embargo, datos bien documentados, aptos para confirmar lo que aquí y allá se ha escrito, es decir, que exista, cuando el tratamiento se prolonga por muchos meses 0 años, un estado similar al de la menopausia. Los conocimientos sobre contraconceptivos orales y "estado pseudogravídico", se refiere principalmente a contraceptivos que con-

TABLA 10

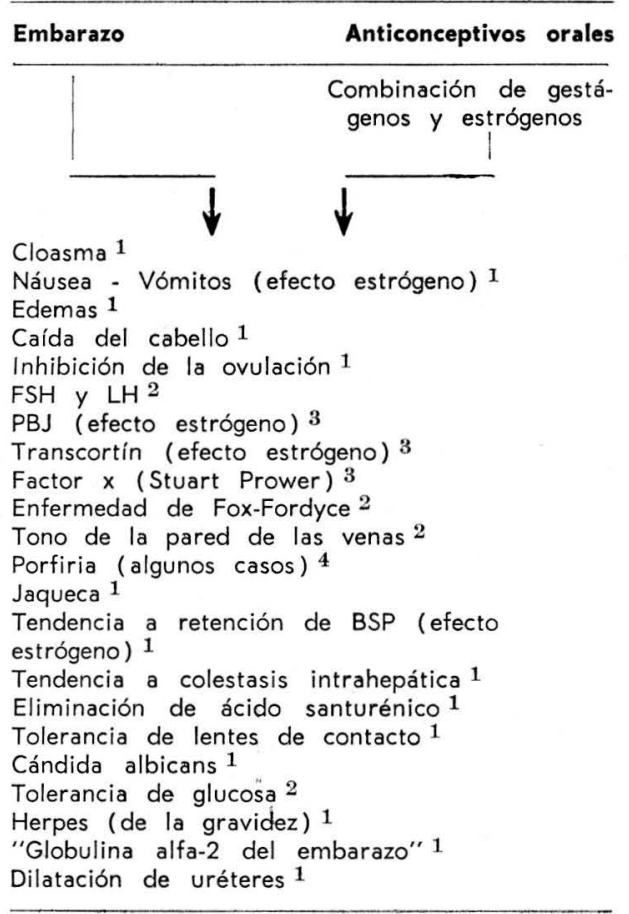

\begin{aligned} & 1$\rightarrow \\ & 2< \\ & 3> \\ & 4> \\ &< \\ &$\hline\end{aligned}

tienen dos o más miligramos de un progestágeno. Cuando dispongamos de mayor conocimiento en temas de modificación bioquímica en la mujer tratada por largo tiempo con combinaciones estro-progestacionales a bajísimas dosis, será interesante revisar de nuevo el punto para ver si con esta cantidad de esteroides hormonales se presentan modificaciones orgánicas $y / 0$ funcionales del tipo menopáusico. Luego de la ingestión de combinaciones estro - progestágenas con fin anticoncepcional, se realiza en efecto, como sabemos, una total - casi total supresión de la secreción hormonal de parte del ovario. En la mujer en edad fecunda - cuyo organismo necesita de una cantidad adecuada de hormonas sexuales- la única fuente de tales hormonas proviene de la píldora anticoncepcional. Una dosis en exceso baja podría, a la larga, crear un estado de insuficiencia relativa.

Ya que estamos en el tema de la contracepción, quisiéramos discutir algunos otros aspectos del problema, aunque de manera muy rápida, dejando al colega Haller la tarea de profundizarlo.

Es notorio que en la actualidad se encuentran en fase de avanzada investigación clínica, respectivamente, una suspensión microcristalina de provera, una suspensión microcristalina de acetato de clormadinona y una solución de una combinación de di-hidroxi-progesterona acetofénica + enantato de estradiol.

Las suspensiones microcristalinas han sido bien estudiadas en Centro y Sur América por Edris Rice-Wray en México y por Zañartu en Chile. La enorme ventaja de su empleo reside no solo en su seguridad sino en el hecho de que se elimina el factor memoria. Introducido el fármaco, el 
sujeto se encuentra en estado de infecundidad por toda la duración del efecto. Esta varía en función de la dosis, pudiendo prolongarse por meses. Existen, no obstante, dos inconvenientes: las hemorragias frecuentes; sean del tipo "break through bleeding", sean del tipo "spotting", y en no raros casos de amenorrea después de finalizar el efecto, en especial con las dosificaciones más altas que son aquellas de duración más prolongada. De la combinación estroprogestógena depot es para mí conocido lo que informa la literatura: con una inyección al mes se obtienen ciclos artificiales más bien regulares, y la contracepción está asegurada.

Se halla en estudio un método completamente nuevo, de implantación sub-cutánea de esteroides progestágenos en un "involucro" de Silastic, una sustancia plástica particular. El método se debe al doctor Sheldon Segal del "Population Council" de Nueva York. Con base en cuanto de él se me ha referido, de la implantación se liberan cantidades mínimas de esteroide suficientes para asegurar una protección contraceptiva adecuada durante meses, y aún de años. $\mathrm{El}$ efecto se interrumpe con la remoción del implanto. Se trata aquí de una "luteal supplementation" de nuevo tipo, que asocia las ventajas de la "suplementation luteal" oral con las de suspensiones microcristalinas intramusculares.

La "luteal supplementation" a la cual apenas nos habíamos referido, merece también una mención ya que - a mi parecer - se trata del más importante de los actuales desarrollos del problema de cómo poner a la disposición del mayor número de personas en todo el mundo un método contraceptivo seguro, simple, de bajo costo y que no inhibe la lac- tancia si el tratamiento se inicia inmediatamente después del parto. Personalmente, gracias a la colaboración de un grupo de colegas, puedo referirme a la suministración ininterrumpida "luteal supplementation" de 0,5 mg. de acetato de clormadinona al día. Los primeros resultados de este tratamiento fueron referidos en Ciudad de México por el grupo de Martínez Manautou. Los datos a mi disposición confirmaron cuanto han publicado los colegas mejicanos, excepción hecha por la observación que cuando el esteroide sea ingerido con regularidad el método tiene casi el mismo índice de seguridad de los clásicos contraceptivos orales compuestos por un estrógeno + un progestágeno. La tolorabilidad es óptima, la lactancia no se ve influída. El exacto mecanismo de acción no está aún del todo claro. Aparece por ahora fuera de toda duda que un papel de primer orden se atribuye a la modificación del moco cervical que presente cierta "hostilidad" a los espermatozoids. En una serie de países cursan cuidadosas investigaciones que aclararán dentro de poco la acción contraceptiva de esta pequeña dosis de clormadinona.

A la terapia anti-estrógena me he referido en forma indirecta, al hablar de la terapia progestagénica. Para usar una frase de Selye, ningún esteroide antagoniza la acción de otro esteroide como una base a un ácido. El efecto estrogénico sobre un receptor dado puede, así mismo, ser modificado en el sentido deseado de ciertos progestágenos. Por ejemplo, el efecto de los estrógenos sobre el endometrio y también sobre el cáncer del endometrio, se puede influir profundamente con una terapia masiva (dosis fármaco-dinámicas) progestógena. Por definición se habla del efecto anti-estrógeno, cuando en rea- 
lidad se trata de una cosa mucho más compleja que llegaremos a definir con precisión solo cuando conozcamos el mecanismo de acción de estos esteroides a nivel celular o hasta "molecular". Con mucho pesar, hasta ahora no existen sustancias antiestrógenas privadas de cualquier otro efecto hormonal.

Cuanto he dicho para los anti-estrógenos, vale también para los antiandrógenos. Existen aquí una serie de sustancias esteroides, que están en estudio, que han sido desarrolladas "ad hoc", o sea, empleadas en presencia de síntomas de hiperandrogenismo - por ejemplo_ en la mujer. Es interesante anotar cómo también con un potente antiandrógeno -los estrógenos- no es posible contrabalancear la sintomatología atribuída por lo general a la presencia de un exceso o a la respuesta exaltando los andrógenos en la mujer. También cuando haya una clara etiología - exceso de testosterona- permanece oscura la patogenia del consecuente complejo sintomático. De otra mane$\mathrm{ra}$, en presencia de un complejo sintomático en apariencia idéntico, no es posible demostrar la existencia de un exceso de andrógenos y la terapia con anti-andrógenos de escasos resultados. Un típico ejemplo de la complejidad de la patología atribuída al hiperandrogenismo en la mujer está representado en el acné, del que hablamos al tratar de los corticoides.
Aquí reina una absoluta "polipragmasia terapéutica". En conclusión, si también dispusiéramos de un antiandrógeno "ideal" sin otro efecto hormonal, no sería, en el estado actual, posible prever los éxitos (o insucesos) terapéuticos que tendríamos en el campo ginecológico.

Ahora, refiriéndome al futuro, inevitablemente debo abandonar los hechos en favor de las suposiciones. En lo que respecta a las contracepciones, los contraceptivos de más fácil aplicación para grandes estratos de la población mundial serían una píldora ingerida una vez al mes. Me imagino un fármaco capaz de interrumpir de un golpe la función del cuerpo lúteo, sea menstrual o gravídico (primer mes antes de la primera amenorrea) que no tenga ninguna influencia sobre la corteza suprarrenal y que actúe sobre el cuerpo lúteo mismo. La súbita deprivación de progesterona provocará una descamación del endometrio. Si el ciclo era anovulatorio, la hemorragia por deprivación de estrógenos no sería influída. Que yo sepa, este fármaco no existe aún. Debo, en este punto, subrayar que hablo como biólogo y que no quiero y no puedo -por incompetencia- discutir los aspectos morales de un fármaco semejante. Falta también, como ya he dicho, un fármaco capaz de influir la contracción uterina, eficaz en la amenaza de un parto prematuro. 